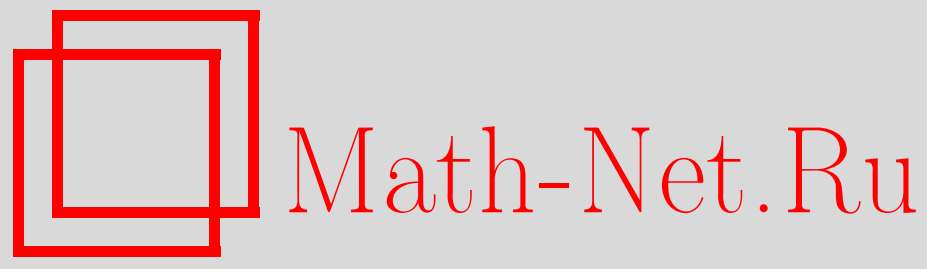

В. А. Лебедев, О существовании слабых решений для стохастических дифференциальных уравнений с ведущими $L^{0}$-значными мерами, Теория вероятн. и ее примен., 2002, том 47, выпуск 4, 672685

DOI: https://doi.org/10.4213/tvp3774

Использование Общероссийского математического портала MathNet.Ru подразумевает, что вы прочитали и согласны с пользовательским соглашением

http://www . mathnet.ru/rus/agreement

Параметры загрузки:

IP : 54.197 .217 .227

26 апреля 2023 г., 13:08:52

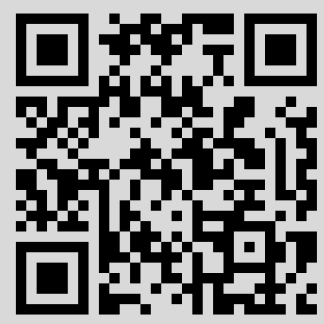




\section{О СУЩЕСТВОВАНИИ СЛАБЫХ РЕШЕНИЙ ДЛЯ СТОХАСТИЧЕСКИХ ДИФФЕРЕНЦИАЛЬНЫХ УРАВНЕНИЙ С ВЕДУЩИМИ $L^{0}$-ЗНАЧНЫМИ МЕРАМИ ${ }^{1)}$}

В настоящей статье для стохастического дифференциального уравнения с $\sigma$-конечной $L^{0}$-значной случайной мерой $\theta$ в смысле Бихтелера и Жакода дается доказательство существования его слабого решения, основанное на подобном результате для частного случая $L^{2}$-значной случайной меры.

Ключевые слова и фразы: $\sigma$-конечная $L^{p}$-значная случайная мера, стохастическое дифференциальное уравнение, слабое решение, расширение стохастического базиса.

1. Введение. Пусть $(\Omega, \mathscr{F}, \mathfrak{F}, \mathbf{P})$ есть стохастический базис, состоящий из вероятностного пространства $(\Omega, \mathscr{F}, \mathbf{P})$ и непрерывной справа фильтрации $\mathfrak{F}=\left(\mathscr{F}_{t}\right)_{t \in \mathbf{R}_{+}}$, и пусть $\mathscr{O}=\mathscr{O}(\mathfrak{F})$ и $\mathscr{P}=\mathscr{P}(\mathfrak{F})-$ соответственно $\mathfrak{F}$-опциональная и $\mathfrak{F}$-предсказуемая $\sigma$-алгебры на $\Omega \times \mathbf{R}_{+}$. Пусть $(E, \mathscr{E})$ есть измеримое пространство.

Напомним определения используемых здесь понятий работы [1], а также [2] и [3]. Прежде всего, $L^{p}(\Omega, \mathscr{F}, \mathbf{P})$ для $p \geqslant 1$ означает банахово пространство интегрируемых в степени $p$ случайных величин $\xi$ (точнее, классов их $\mathbf{P}$-эквивалентности) с нормой $\|\xi\|_{p}=\left(\mathbf{E}|\xi|^{p}\right)^{1 / p}$, а $L^{p}(\Omega, \mathscr{F}, \mathbf{P})$ для $0 \leqslant p<1$ есть $F$-пространство интегрируемых в степени $p$ случайных величин $\xi$ с квазинормой $\|\xi\|_{p}=\mathbf{E}\left(|\xi| \wedge|\xi|^{p}\right)$. В частности, $L^{0}(\Omega, \mathscr{F}, \mathbf{P})$ содержит все случайные величины на вероятностном пространстве $(\Omega, \mathscr{F}, \mathbf{P})$, и сходимость в нем совпадает со сходимостью по вероятности.

Основным понятием работ [1]-[3] является $\sigma$-конечная $L^{p}$-значная для $p \geqslant 0$ случайная мера на $\left(\Omega \times \mathbf{R}_{+} \times E, \mathscr{P} \otimes \mathscr{E}\right)$, а именно семейство $\theta=\left(\theta_{t}\right)_{t \in \mathbf{R}_{+}}$, удовлетворяющее следующим условиям:

а) $\theta_{t}$ для каждого $t \in \mathbf{R}_{+}$есть $\sigma$-конечная мера на $\left(\Omega \times \mathbf{R}_{+} \times E, \mathscr{P} \otimes \mathscr{E}\right)$ со значениями в $L^{p}\left(\Omega, \mathscr{F}_{t}, \mathbf{P}\right)$, т.е. имеется такая строго положительная

${ }^{*}$ Московский государственный университет им. М. В. Ломоносова, механико-математический факультет, лаборатория математической статистики, Ленинские горы, 119992 Москва, Россия.

${ }^{1}$ Работа выполнена при поддержке Российского фонда фундаментальных исследований, проект № 96-15-96033. 
$\mathscr{P} \otimes \mathscr{E}$-измеримая функция $V$ на $\Omega \times \mathbf{R}_{+} \times E$, что если $(\mathscr{P} \otimes \mathscr{E})_{V}=$ $\{\varphi: \varphi \mathscr{P} \otimes \mathscr{E}$-измеримы, $\varphi / V$ ограничены $\}$, то мы имеем:

(а-1) $\theta_{t}$ - линейное отображение из $(\mathscr{P} \otimes \mathscr{E})_{V}$ в $L^{p}\left(\Omega, \mathscr{F}_{t}, \mathbf{P}\right)$,

$\left(\right.$ а-2) если $\left(\varphi_{n}\right)$ - последовательность в $(\mathscr{P} \otimes \mathscr{E})_{V}$ с $\left|\varphi_{n}\right| \leqslant V$, сходящаяся поточечно к 0 , то $\theta_{t}\left(\varphi_{n}\right) \rightarrow 0$ в $L^{p}\left(\Omega, \mathscr{F}_{t}, \mathbf{P}\right)$;

б) $\theta_{s}(\varphi)=\theta_{t}\left(\varphi 1_{[0, s]}\right)$ для всех $\varphi \in(\mathscr{P} \otimes \mathscr{E})_{V}$ и $s \leqslant t$;

в) $\theta_{t}\left(\varphi 1_{A \times I \times E}\right)=1_{A} \theta_{t}\left(\varphi 1_{\Omega \times I \times E}\right)$ для всех $\varphi \in(\mathscr{P} \otimes \mathscr{E})_{V}$ и $t \in \mathbf{R}_{+}$, если $A \in \mathscr{F}_{0}$ и $I=\mathbf{R}_{+}$или если $A \in \mathscr{F}_{s}$ и $\left.\left.I=\right] s, s^{\prime}\right]$ с $s<s^{\prime}$.

В частности, когда $V \equiv 1, \theta$ называется конечной $L^{p}$-значной случайной мерой. Обозначим через $\widetilde{\mathscr{S}}_{\sigma}^{p}\left(\right.$ соответственно $\left.\widetilde{\mathscr{S}}^{p}\right)$ пространство всех $\sigma$-конечных (конечных) $L^{p}$-значных случайных мер на $\left(\Omega \times \mathbf{R}_{+} \times\right.$ $E, \mathscr{P} \otimes \mathscr{E})$.

Мы полагаем для $\theta \in \widetilde{\mathscr{S}}_{\sigma}^{p}$

$$
\|\varphi\|_{L^{1, p}(\theta)}=\sum_{N=1}^{\infty} 2^{-N}\left(1 \bigwedge \sup _{\substack{\psi \in(\mathscr{S} \otimes \mathcal{E})_{V} \\|\psi| \leqslant|\varphi|}}\left\|\theta_{N}(\psi)\right\|_{p}\right)
$$

и обозначаем через $L^{1, p}(\theta)$ множество $\mathscr{P} \otimes \mathscr{E}$-измеримых функций $\varphi$, для каждой из которых имеется последовательность $\left(\varphi_{n}\right) \subset(\mathscr{P} \otimes \mathscr{E})_{V}$ с $\left\|\varphi_{n}-\varphi\right\|_{L^{1, p}(\theta)} \rightarrow 0$. Тогда для каждого $t \in \mathbf{R}_{+} \theta_{t}\left(\varphi_{n}\right)$ стремится к пределу в $L^{p}\left(\Omega, \mathscr{F}_{t}, \mathbf{P}\right)$, который не зависит от выбора $\left(\varphi_{n}\right)$ для данной $\varphi$ и обозначается $\theta_{t}(\varphi)$. При этом если $p \leqslant q$ и $\theta \in \widetilde{\mathscr{S}}_{\sigma}^{q}$, то $\theta \in \widetilde{\mathscr{S}}_{\sigma}^{p}$ и $L^{1, q}(\theta) \subset$ $L^{1, p}(\theta)$. Относительно квазинормы $\|\cdot\|_{L^{1, p}(\theta)}$ пространство $L^{1, p}(\theta)$ полно.

Аналогично на пространстве $\widetilde{\mathscr{S}}^{p}$ конечных случайных мер можно ввести квазинорму

$$
\|\theta\|_{\tilde{\mathscr{S}}_{p}}=\sum_{N=1}^{\infty} 2^{-N}\left(1 \bigwedge \sup _{\substack{\varphi \in(\mathscr{P} \otimes \mathcal{S})_{1} \\|\varphi| \leqslant 1}}\left\|\theta_{N}(\varphi)\right\|_{p}\right),
$$

и тогда $\widetilde{\mathscr{S}}^{p}$ полно относительно этой квазинормы.

Приведем теперь пример, важный для дальнейшего развития теории в целом. Именно, пусть $E=\{1\}$, т.е. состоит из одной точки, так что мы рассматриваем случайные меры на $\left(\Omega \times \mathbf{R}_{+}, \mathscr{P}\right)$. Тогда по теореме Деллашери-Мокободского-Бихтелера (см. [1, теорема 1.1]) имеется взаимно однозначное соответствие между множеством конечных $L^{0}$ значных случайных мер на $\left(\Omega \times \mathbf{R}_{+}, \mathscr{P}\right)$ и множеством семимартингалов, определяемых, с точностью до неотличимости, по формуле

$$
\theta_{t}(H)=H \cdot X_{t}
$$

для всякого ограниченного предсказуемого $H$ при каждом $t \in \mathbf{R}_{+}$. Что касается $\sigma$-конечных $L^{0}$-значных случайных мер, то они обычно называются формальными семимартингалами. Обозначая через $\mathscr{S}^{p}$ и $\mathscr{S}_{\sigma}^{p}$ соответственно $\widetilde{\mathscr{S}}^{p}$ и $\widetilde{\mathscr{S}}_{\sigma}^{p}$ для $E=\{1\}$, заметим, что относительно соответствующей квазинормы топология на $\mathscr{S}^{0}$ совпадает с топологией 
Эмери [4] на пространстве семимартингалов, а на $\mathscr{S}^{p}$ для $p \geqslant 1$ - с топологией сходимости в $\mathscr{H}^{p}$ из [4] на каждом конечном интервале.

Пусть $\theta \in \widetilde{\mathscr{S}}_{\sigma}^{p}$ и $\varphi \in L^{1, p}(\theta)$. Тогда равенство

$$
(\varphi \times \theta)_{t}(H)=\theta_{t}(H \varphi)
$$

для $H \in \mathscr{P}_{1}$ определяет семейство $\varphi \times \theta$ как конечную $L^{p}$-значную меру на $\left(\Omega \times \mathbf{R}_{+}, \mathscr{P}\right)$, для которой в силу предыдушего имеется семимартингал, обозначаемый также $\varphi \times \theta$ и называемый прочессом стохастического интеграла для $\varphi$ относительно $\theta$, и в силу (1)

$$
\varphi \times \theta_{t}=(\varphi \times \theta)_{t}(1)=\theta_{t}(\varphi) .
$$

Можно определить интегралы относительно $\theta \in \widetilde{\mathscr{S}}_{\sigma}^{p}$ для более широкого класса $\mathscr{P} \otimes \mathscr{E}$-измеримых функций, чем $L^{1, p}(\theta)$. Введем множество $L_{\sigma}^{p}(\theta)=\{\varphi: \varphi \mathscr{P} \otimes \mathscr{E}$-измеримы и имеется строго положительный предсказуемый процесс $K$ такой, что $\left.K \varphi \in L^{1, p}(\theta)\right\}$. Тогда равенство (2) для предсказуемых $H$ с ограниченными $H / K$ определяет $\varphi \times \theta$ как $\sigma$ конечную $L^{p}$-значную случайную меру на $\left(\Omega \times \mathbf{R}_{+}, \mathscr{P}\right)$, т.е. как формальный семимартингал. Теперь выделим множество $\widehat{L}^{p}(\theta) \subset L_{\sigma}^{p}(\theta)$ таких $\varphi$, для которых $\varphi \times \theta-$ конечная $L^{p}$-значная случайная мера на $\left(\Omega \times \mathbf{R}_{+}, \mathscr{P}\right)$, т.е. семимартингал. Очевидно, $L^{1, p}(\theta) \subset \widehat{L}^{p}(\theta)$ и $\widehat{L}^{q}(\theta) \subset \widehat{L}^{p}(\theta)$ для $p \leqslant q$.

В частности, если $p=2$, а $(E, \mathscr{E})$ - лузинское пространство с борелевской $\sigma$-алгеброй, то, согласно теореме 1 из [2], существуют предсказуемый возрастающий процесс $X^{1}$, начинающиеся в 0 квадратично интегрируемые мартингалы $X^{i}$ для $i \geqslant 2$ с $\left\langle X^{i}, X^{j}\right\rangle \equiv 0$ для $i \neq j$ и регулярные (знакопеременные) переходные меры $\rho^{i}$ из $\left(\Omega \times \mathbf{R}_{+}, \mathscr{P}\right)$ в $(E, \mathscr{E})$ такие, что для всякой $\varphi \in \widehat{L}^{2}(\theta)$ ее сечения $\rho^{i}$-интегрируемы почти всюду соответственно по мерам $\mathbf{P} \times d X^{1}$ и $\mathbf{P} \times d\left\langle X^{i}, X^{i}\right\rangle$ для $i \geqslant 2$, и тогда

$$
\varphi \times \theta=\sum_{i=1}^{\infty} \int_{E} \varphi \rho_{s}^{i}(\omega, d u) \cdot X^{i},
$$

где в правой части стоит ряд из процессов стохастических интегралов по семимартингалам $X^{i}$, сходящийся безусловно в $\mathscr{S}^{2}$. Кроме того, мы выбираем мартингалы $X^{i}$ для $i=3 k-1$ непрерывными, для $i=3 k-$ чисто разрывными и квазинепрерывными слева, а для $i=3 k+1$ - чисто разрывными и достижимыми. Пусть $B$ есть некоторый предсказуемый возрастающий процесс, относительно которого абсолютно непрерывны $X^{1}$ и $\left\langle X^{i}, X^{i}\right\rangle$ для всех $i \geqslant 2$. Именно в этих терминах формулируется результат о существовании слабого решения уравнения с мерой $\theta$ для $p=2$.

Заметим также, что к этому случаю сводится и более общий случай, когда $(E, \mathscr{E})$ - сепарабельное метрическое пространство, универсально измеримое в своем пополнении, с борелевской $\sigma$-алгеброй. 
Именно, согласно предложению 1 из [5], существует неотрицательная доминируюшая мера $\mu$ для $\theta$ на $\left(\Omega \times \mathbf{R}_{+} \times E, \mathscr{P} \otimes \mathscr{E}\right)$, и тогда существует $\sigma$-компактное множество $\widetilde{E} \subset E$, на котором сосредоточен $E$-маргинал меры $\mu$. Сама мера $\mu$ тогда сосредоточена на множестве $\left(\Omega \times \mathbf{R}_{+} \times \widetilde{E}, \mathscr{P} \otimes \widetilde{\mathscr{E}}\right)$, где $\widetilde{\mathscr{E}}-$ след $\sigma$-алгебры $\mathscr{E}$ на $\widetilde{E}$, и $\theta$ можно рассматривать как $\sigma$-конечную $L^{2}$-значную случайную меру на $\left(\Omega \times \mathbf{R}_{+} \times \widetilde{E}, \mathscr{P} \otimes \widetilde{\mathscr{E}}\right)$, так как для любой $\varphi \in \widehat{L}^{2}(\theta)$ стохастический интеграл $\varphi \times \theta$ не зависит от значений функции $\varphi$ на $\Omega \times \mathbf{R}_{+} \times(E \backslash \widetilde{E})$.

Для формулировки более общего результата с $L^{0}$-значной случайной мерой понадобятся следующие определения, обозначения и факты. Именно, пусть $W$ есть $\mathscr{O} \otimes \mathscr{E}$-измеримая функция на $\Omega \times \mathbf{R}_{+} \times E$ вида $W=W_{1}+W_{2} 1_{D \times E}$, где $W_{1}$ и $W_{2}-\mathscr{P} \otimes \mathscr{E}$-измеримые функции, а $D$ дискретное опциональное случайное множество. Если $\theta \in \widetilde{\mathscr{S}}_{\sigma}^{0}$, то можно определить меру $W \cdot \theta \in \widetilde{\mathscr{S}}_{\sigma}^{0}$ следующим образом. Именно, для $\mathscr{P} \otimes \mathscr{E}$-измеримой функции $\varphi$ на $\Omega \times \mathbf{R}_{+} \times E$ такой, что $W_{1} \varphi$ и $W_{2} \varphi$ принадлежат $L^{1,0}(\theta)$, определим

$$
(W \cdot \theta)_{t}(\varphi)=\theta_{t}\left(W_{1} \varphi\right)+\sum_{i=1}^{\infty} \Delta\left(W_{2} \varphi \times \theta\right)_{\tau_{i}} 1_{\left\{\tau_{i} \leqslant t\right\}}
$$

где $\left(\tau_{i}\right)_{i \in \mathrm{N}}$ - возрастающая последовательность моментов остановки, графики которых составляют множество $D$. В силу предложения 4.18 из [1] это не зависит от выбора представления функции $W$. В частности, если $\theta \in \widetilde{\mathscr{S}}_{\sigma}^{p}$ для некоторого $p \geqslant 1$, а $W_{1}, W_{2} \in L^{1, p}(\theta)$, то $W \cdot \theta \in \widetilde{\mathscr{S}}_{\sigma}^{p}$. В этих терминах оказывается верным следующий результат, представляющий собой теорему 2 из [6], для которого мы дадим более краткое и точное доказательство.

Предложение. Пусть $\theta-\sigma$-конечная $L^{0}$-значная случайная мера на $\left(\Omega \times \mathbf{R}_{+} \times E, \mathscr{P} \otimes \mathscr{E}\right)$. Тогда $\theta$ является $L^{2}$-значной случайной мерой предлокально, т.е. для всякой функиии $\varphi \in \widehat{L}^{0}(\theta)$ существует $\mathbf{P}-n . н$. возрастающая $\kappa$ бесконечности последовательность $\left(\tau_{n}\right)_{n \in \mathbf{N}}$ моментов остановки такая, что $\varphi \in \widehat{L}^{2}\left(1_{\left[0, \tau_{n}[\times E\right.} \cdot \theta\right)$ для всех $n \in \mathbb{N}$.

Д ок а $з$ а т е л ь с т в о. Пусть $\varphi \in \widehat{L}^{0}(\theta)$. Тогда существует строго положительный $\mathscr{P}$-измеримый процесс $K$ такой, что $K \varphi$ принадлежит $L^{1,0}(\theta)$, и если $V-$ некоторая строго положительная $\mathscr{P} \otimes \mathscr{E}$ измеримая функция на $\Omega \times \mathbf{R}_{+} \times E$, принадлежащая $L^{1,0}(\theta)$, то, согласно теореме 2 из [2], существует вероятностная мера $\mathbf{Q}$ на $(\Omega, \mathscr{F})$, эквивалентная $\mathbf{P}$, с ограниченной производной $Z_{\infty}=d \mathbf{Q} / d \mathbf{P}$, такая, что $W=K|\varphi|+V$ принадлежит $L^{1,2}(\theta, \mathbf{Q})$.

Теперь определим моменты остановки

$$
\tau_{n}=\inf \left\{t \in \mathbb{R}_{+}: Z_{t} \leqslant \frac{1}{n}\right\}
$$


где $Z$ - càdlàg (т.е. с непрерывными справа и имеющими пределы слева траекториями) мартингал, $Z_{t}=\mathbf{E}\left(Z_{\infty} \mid \mathscr{F}_{t}\right)$. Для фиксированного $n$ заметим прежде всего, что если $B$ - согласованный неотрицательный возрастающий процесс, не имеющий скачка в момент $\tau_{n}$, то

$$
\mathbf{E} B_{\tau_{n}} \leqslant n \mathbf{E}_{\mathbf{Q}} B_{\tau_{n}}
$$

где $\mathbf{E}_{\mathbf{Q}}$ - математическое ожидание по мере Q. Действительно, по теореме 47 из $[7$, гл. IV] имеем

$$
\begin{aligned}
\mathbf{E} B_{\tau_{n}} & =\mathbf{E}_{\mathbf{Q}} \frac{B_{\tau_{n}}}{Z_{\tau_{n}}}=\mathbf{E}_{\mathbf{Q}} \int_{\left[0, \tau_{n}\right]} \frac{d B_{t}}{Z_{t}}=\mathbf{E}_{\mathbf{Q}} \int_{\left[0, \tau_{n}\right]} \frac{1_{\left\{t<\tau_{n}\right\}} d B_{t}}{Z_{t}} \\
& \leqslant \mathbf{E}_{\mathbf{Q}} \int_{\left[0, \tau_{n}\right]} n d B_{t}=n \mathbf{E}_{\mathbf{Q}} B_{\tau_{n}} .
\end{aligned}
$$

Пусть $|\psi| \leqslant W$. Тогда в силу (4)

$$
\mathbf{E} \sup _{0 \leqslant s \leqslant t}\left[(\psi \times \theta)_{s}^{\tau_{n}-}\right]^{2} \leqslant n \mathbf{E}_{\mathbf{Q}} \sup _{0 \leqslant s \leqslant t}\left[(\psi \times \theta)_{s}^{\tau_{n}-}\right]^{2} \leqslant n \mathbf{E}_{\mathbf{Q}} \sup _{0 \leqslant s \leqslant t}\left[(\psi \times \theta)_{s}\right]^{2},
$$

так что $1_{\llbracket 0, \tau_{n} \llbracket \times E} \cdot \theta \in \widetilde{\mathscr{S}}_{\sigma}^{2}, W \in L^{1,2}\left(1_{\left[0, \tau_{n} \llbracket \times E\right.} \cdot \theta\right)$ и $\varphi \in \widehat{L}^{2}\left(1_{\left[0, \tau_{n} \llbracket \times E\right.} \cdot \theta\right)$ для любого $n \in \mathbf{N}$, что и требовалось.

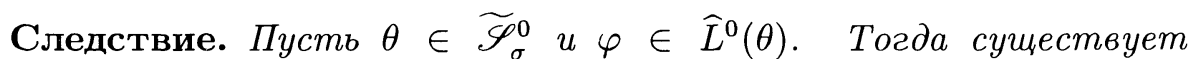
дискретное опциональное случайное множество $D_{0}$ такое, что мера $\tilde{\theta}=1_{D^{c} \times E} \cdot \theta$ принадлежит $\widetilde{\mathscr{S}}_{\sigma}^{2}$ для любого дискретного опиионального множества $D, D \supset D_{0}, u$ тогда $\varphi \in L_{\sigma}^{2}(\widetilde{\theta})$.

Д о к а з а т ел ь с т в о. Применим предложение к $\theta$ и $\varphi$, и пусть $D_{0}=\bigcup_{n=1}^{\infty}\left[\llbracket \tau_{n} \rrbracket\right.$. Так как $D$ есть объединение графиков моментов остановки, также удовлетворяющих заключению предложения, то можно считать, что $D=D_{0}$. Применяя предложение 4.18 из [1], легко видеть, что для каждого $n \in \mathbf{N}$ имеет место $1_{\left(\left[0, \tau_{n}\left[\cap D^{c}\right) \times E\right.\right.} \cdot \theta \in \mathscr{S}_{\sigma}^{2}$ и

$$
\varphi \in \widehat{L}^{2}\left(1_{\left(\left[0, \tau_{n}\left[\cap D^{c}\right) \times E\right.\right.} \cdot \theta\right) .
$$

Но $\llbracket 0, \tau_{n} \llbracket \cap D^{c}=\llbracket 0, \tau_{n} \rrbracket \cap D^{c}$, откуда легко получается требуемое.

2. Основные результаты. Пусть теперь $\check{\Omega}$ есть множество всех $\mathbf{R}^{d}$-значных функций на $\mathbf{R}_{+}, \check{\mathscr{F}}$ - его борелевская $\sigma$-алгебра для тихоновской топологии, $\breve{\mathfrak{F}}$ - фильтрация из $\sigma$-алгебр $\check{\mathscr{F}}_{t}$ для $t \in \mathbf{R}_{+}$, каждая из которых есть пересечение для $s>t \sigma$-подалгебр из $\mathscr{F}$, порождаемых сужениями на $[0, s]$ функций из $\check{\Omega}$. Пусть тогда $X$ есть канонический процесс на $\check{\Omega}$, т.е. $X_{t}(\check{\omega})=\check{\omega}_{t}$. Пусть также $\bar{\Omega}=\Omega \times \check{\Omega}, \overline{\mathscr{F}}=\mathscr{F} \otimes \check{\mathscr{F}}$, $\overline{\mathscr{F}}_{t}=\bigcap_{s>t}\left(\mathscr{F}_{s} \otimes \check{\mathscr{F}}_{s}\right)$ и $\overline{\mathfrak{F}}=\left(\overline{\mathscr{F}}_{t}\right)_{t \in \mathbf{R}_{+}}$.

Рассмотрим $\mathscr{P}(\overline{\mathfrak{F}}) \otimes \mathscr{E}$-измеримую $d$-мерную вектор-функцию $h$ на $\bar{\Omega} \times \mathbf{R}_{+} \times E$ и уравнение

$$
X=N+h(X) \times \theta
$$


для $\theta \in \widetilde{\mathscr{S}}_{\sigma}^{0}, \mathbf{P}$-п.н. не нагружающей множество $\{0\} \times E$, где $N$ есть заданный $\mathbf{R}^{d}$-значный $\mathfrak{F}$-прогрессивно измеримый процесс, играющий роль начального условия. Тогда как в [3], аналогично [8], [9] и [10], можно определить слабое решение уравнения (5). Именно, решаюшая мера (или слабое решение) уравнения (5) есть такая вероятностная мера $\overline{\mathbf{P}}$ на $(\bar{\Omega}, \overline{\mathscr{F}})$, что ее $\Omega$-маргинал $\left.\overline{\mathbf{P}}\right|_{\Omega}$ равен $\mathbf{P}$, уравнение $(5)$ сохраняет свой смысл на $(\bar{\Omega}, \overline{\mathscr{F}}, \overline{\mathfrak{F}}, \overline{\mathbf{P}})$ и канонический процесс $X$ является решающим процессом уравнения (5). Сохранение смысла уравнением (5) означает выполнение следующих двух условий:

а) $\theta$ допускает продолжение на $\left(\bar{\Omega} \times \mathbf{R}_{+} \times E, \mathscr{P}(\overline{\mathfrak{F}}) \otimes \mathscr{E}\right)$ как $\sigma$-конечная $L^{0}(\bar{\Omega}, \overline{\mathscr{F}}, \overline{\mathbf{P}})$-значная случайная мера, т.е. существует строго положительная $\mathscr{P}(\mathfrak{F}) \otimes \mathscr{E}$-измеримая функция $V$ на $\Omega \times \mathbf{R}_{+} \times E$, принадлежашая $L^{1,0}(\theta, \overline{\mathfrak{F}})$ и такая, что для всех $\mathscr{P}(\mathfrak{F}) \otimes \mathscr{E}$-измеримых $\varphi$ на $\Omega \times \mathbf{R}_{+} \times E$ с $|\varphi| \leqslant V$ процессы стохастического интеграла $\varphi \times \theta$ на $\mathfrak{F}$ и $\overline{\mathfrak{F}}$ определены и совпадают (тем самым, очевидно, $V \in L^{1,0}(\theta, \mathfrak{F})$ );

б) если $\mathscr{P}(\mathfrak{F}) \otimes \mathscr{E}$-измеримая функция $\varphi$ на $\Omega \times \mathbf{R}_{+} \times E$ принадлежит $\widehat{L}^{0}(\theta, \overline{\mathfrak{F}})$, то она принадлежит и $\widehat{L}^{0}(\theta, \mathfrak{F})$, и, кроме того, процессы стохастического интеграла $\varphi \times \theta$ на $\mathfrak{F}$ и $\overline{\mathfrak{F}}$ также совпадают.

Согласно теореме 3 из [2], условия а) и б) выполняются, в частности, когда $(\bar{\Omega}, \bar{F}, \overline{\mathfrak{F}}, \overline{\mathbf{P}})$ является очень хорошим расширением стохастического базиса $(\Omega, \mathscr{F}, \mathfrak{F}, \mathbf{P})$, т.е. когда всякий $\mathfrak{F}$-мартингал является $\overline{\mathfrak{F}}$-мартингалом. В этом случае сама решающая мера также называется очень хорошей.

Сформулируем теорему существования слабого решения для уравнения (5) с $L^{2}$-значной случайной мерой $\theta$.

Теорема 1. Пусть $\theta \in \mathscr{S}_{\sigma}^{2},(E, \mathscr{E})-$ сепарабельное метрическое пространство, универсально измеримое в своем пополнении, с его борелевской $\sigma$-алгеброй, и для уравнения (5) выполнень следуюшие предположения:

1) справедливо неравенство

$$
\left|\int_{E} h \rho^{1}(d u)\right| \frac{d X^{1}}{d B}+\sum_{i=2}^{\infty}\left|\int_{E} h \rho^{i}(d u)\right|^{2} \frac{d\left\langle X^{i}, X^{i}\right\rangle}{d B} \leqslant c
$$

$\mathbf{P} \times d B$-n.в. на $\Omega \times \mathbf{R}_{+}$при всех $\check{\omega} \in \check{\Omega}$ для $\mathscr{P}(\mathfrak{F})$-измеримого проиесса $c$, имеюшего $\mathbf{P}$-п.н. конечный для любого $t \in \mathbf{R}_{+}$интеграл $c \cdot B_{t}$;

2) Функции $\int_{E} h \rho^{i}(d u)$ непрерьвны по $\check{\omega} \in \check{\Omega}$ в $U$-топологии почти всюду соответственно по мерам $\mathbf{P} \times d X^{1}$ u $\mathbf{P} \times d\left\langle X^{i}, X^{i}\right\rangle$ для $i \geqslant 2$ на $\Omega \times \mathbf{R}_{+}, u \mathbf{P} \times d B-$ n.в. на $\Omega \times \mathbf{R}_{+}$pяды

$$
\sum_{k=1}^{\infty}\left|\int_{E} h \rho^{3 k-1}(d u)\right|^{2} \frac{d\left\langle X^{3 k-1}, X^{3 k-1}\right\rangle}{d B} \quad u \sum_{k=1}^{\infty}\left|\int_{E} h \rho^{3 k}(d u)\right|^{2} \frac{d\left\langle X^{3 k}, X^{3 k}\right\rangle}{d B}
$$

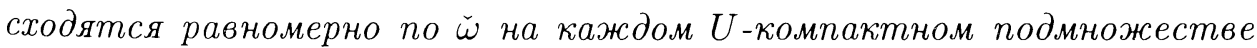

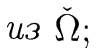


3) если н есть иелочисленная случайная мерана $\left(\mathbf{R}_{+} \times \mathbf{R}^{\mathbf{N}}, \mathscr{B}\left(\mathbf{R}_{+}\right)\right.$ $\left.\otimes \mathscr{B}\left(\mathbf{R}^{\mathbf{N}}\right)\right)$, порождаемая скачками прочесса $\left(X^{3}, \ldots, X^{3 k}, \ldots\right)($ m.е. его мера скачков), с дуальной предсказуемой проекцией $\nu$, то функиия

$$
\sum_{k=1}^{\infty} x_{k}^{2}\left|\int_{E} h \rho^{3 k}(d u)\right|^{2}
$$

$\nu$-интегрируема на $\rrbracket 0, \tau \rrbracket \times \mathbf{R}^{\mathbf{N}}$ для любого $\mathfrak{F}$-момента остановки $\tau$ c

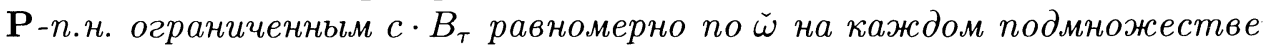
из $\check{\Omega}$ вида $N(\omega)+K$, где $K$ компактно для $J_{1}$-топологии.

Тогда для уравнения (5) существует очень хорошая решаюшая мера.

Теорема является незначительным обобщением теоремы из [3], и в целом ее доказательство проходит аналогично теореме 1 из [9] или теореме 9.20 из [10]. Заметим лишь, что в пункте 2) условия теоремы 1 равномерная по $\check{\omega}$ сходимость рядов на $U$-компактных множествах из $\check{\Omega}$ эквивалентна вместе с непрерывностью функций $\int_{E} h \rho^{i}(d u)$ в $U$-топологии непрерывности в этой топологии по $\check{\omega} \in \check{\Omega}$ сумм этих рядов, используемой в доказательстве теоремы. Условие же 3) теоремы 1 обеспечивает плотное мажорирование скачков для последовательных приближений к слабому решению уравнения (5) и выполняется, в частности, в условиях теоремы из [3], где $\int_{E} h \rho^{3 k}(d u)$ ограничены по $\check{~ в ~ с и л у ~}$ условия 1) теоремы 1 , а ряд

$$
\sum_{k=1}^{\infty}\left|\int_{E} h \rho^{3 k}(d u)\right|^{2} \frac{d\left\langle X^{3 k}, X^{3 k}\right\rangle}{d B}
$$

сходится равномерно по $\check{~ н а ~ с о о т в е т с т в у ю щ е м ~ м н о ж е с т в е, ~ н о ~ о б р а т н о е, ~}$ вообще говоря, неверно.

Действительно, пусть, например, $X$ есть стохастически непрерывный однородный случайный процесс с независимыми приращениями вида $X=u * \mu$, где $\mu-$ мера его скачков с дуальной предсказуемой проекцией $\nu(d s \times d u)=1_{[0,1]}(u) d s d u$ (т.е. его мера Леви сосредоточена на ]0,1] и совпадает на этом интервале с лебеговой мерой), и $\theta=\mu$ с $(E, \mathscr{E})=(\mathbf{R}, \mathscr{B}(\mathbf{R}))$. Тогда в качестве $X^{1}$ в разложении $(3)$ можно взять $X_{t}^{1}=t$, в качестве $X^{3 k}$ - процессы $H_{k}(u) *(\mu-\nu)$, где $H_{k}-k$-я функция Хаара (см. [11, гл. II, $\S 11])$, доопределенная нулем вне ]0, 1], в качестве $B$ процесс $B_{t}=t$, так что

$$
\rho^{1}(d u)=1_{j 0,1]}(u) d u=H_{1}(u) d u, \quad \rho^{3 k}(d u)=\frac{H_{k}(u) d u}{\int_{0}^{1} H_{k}^{2}(v) d v}=H_{k}(u) d u
$$

а для остальных $i$ можно взять $X^{i}$ и $\rho^{i}$ равными нулю. Если теперь $\widetilde{\nu}$ - дуальная предсказуемая проекция меры скачков процесса $\left(X, X^{3}, \ldots, X^{3 k}, \ldots\right)$ со значениями в $\mathbf{R}^{\mathbf{N}}$, а $\left(x_{0}, x_{1}, \ldots, x_{k}, \ldots\right)-$ соответствующие координатные отображения из $\mathbf{R}^{\mathbf{N}}$ в $\mathbf{R}$, то она имеет вид 
$\widetilde{\nu}(d s \times d x)=d s \widetilde{\rho}(d x)$, где $\widetilde{\rho}$ сосредоточена на множестве точек вида $\left(x_{0}, H_{1}\left(x_{0}\right), \ldots, H_{k}\left(x_{0}\right), \ldots\right)$ для $\left.\left.x_{0} \in\right] 0,1\right]$, и ее $x_{0}$-маргинал является лебеговой мерой на $] 0,1]$. Пусть теперь $R_{n}-n$-я функция Радемахера. Тогда $R_{n}(u)=2^{-(n-1) / 2} \sum_{k=2^{n-1}+1}^{2^{n}} H_{k}(u)$, и

$$
\begin{aligned}
\sum_{k=1}^{\infty} x_{k}^{2}\left(\int_{0}^{1} R_{n}(u) \rho^{3 k}(d u)\right)^{2} & =\sum_{k=1}^{\infty} x_{k}^{2}\left(\int_{0}^{1} R_{n}(u) H_{k}(u) d u\right)^{2} \\
& =2^{-(n-1)} \sum_{k=2^{n-1}+1}^{2^{n}} x_{k}^{2}
\end{aligned}
$$

и на множестве, где сосредоточена мера $\widetilde{\rho}$, это равно просто 1 , что равномерно по $n$ интегрируемо по конечной мере $\widetilde{\rho}$ и, следовательно, по $\widetilde{\nu}$ на $] 0, t] \times \mathbf{R}$ для любого конечного $t \in \mathbf{R}_{+}$и тем самым по ее $\left(t, x_{1}, \ldots, x_{k}, \ldots\right)$-маргинальной проекции. Но ряд

$$
\sum_{k=1}^{\infty}\left(\int_{0}^{1} R_{n}(u) H_{k}(u) d u\right)^{2}
$$

сходится по $n$ неравномерно, так как его отличные от нуля члены имеют индексы $2^{n-1}<k \leqslant 2^{n}$.

Сформулируем теперь основной результат этой статьи, относящийся к уравнению (5) с $L^{0}$-значной случайной мерой и незначительно обобщающий результат, анонсированный в [12].

Теорема 2. Пусть $\theta \in \mathscr{S}_{\sigma}^{0},(E, \mathscr{E})$ - сепарабельное метрическое пространство, универсально измеримое в своем пополнении, с его боре-

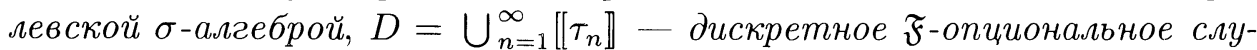
чайное множество, для которого $1_{D^{c} \times E} \cdot \theta \in \widetilde{\mathscr{S}}_{\sigma}^{2}$, и для каждого $n \in \mathbf{N}$ существует такая строго положительная $\mathscr{F}_{\tau_{n}}$-измеримая случайная величина $g_{n}$, что $g_{n} 1_{\llbracket \tau_{n} \rrbracket \times E} \cdot \theta \in \widetilde{\mathscr{S}}_{\sigma}^{2}$, причем на каждом множестве вида $\rrbracket \tau_{n-1}, \tau_{n} \rrbracket \times E$ с $\tau_{0}=0$ условия теоремь 1 выполняются для функиии $h$ имер $1_{D^{c} \times E} \cdot \theta$ u $g_{n} 1_{\llbracket \tau_{n} \rrbracket \times E} \cdot \theta$ вместо $\theta$. Тогда для уравнения (5) существует очень хорошая решающая мера.

Заметим, что последовательность $\left(\tau_{n}\right)$ в теореме необязательно брать $\mathbf{P}$-п.н. строго возрастающей.

Для доказательства теоремы 2 потребуется следующая спецификация определения расширения стохастического базиса, фигурирующего в [8] и [10]. Именно, пусть $(\Omega, \mathscr{F}, \mathfrak{F}, \mathbf{P})-$ стохастический базис с непрерывной справа фильтрацией $\mathfrak{F}=\left(\mathscr{F}_{t}\right)_{t \in \mathbf{R}_{+}}$и $\mathscr{F}_{\infty}=\bigvee_{t \in \mathbf{R}_{+}} \mathscr{F}_{t}$, а $T$ - момент остановки относительно него. Пусть $\widetilde{T}_{t}=(t-T) \vee 0$, тогда для всякого $t \in \mathbf{R}_{+} \tilde{T}_{t}$ является моментом остановки относительно $(\Omega, \mathscr{F}, \widetilde{\mathfrak{F}}, \mathbf{P})$ с $\widetilde{\mathfrak{F}}=\left(\widetilde{\mathscr{F}}_{t}\right)_{t \in \mathbf{R}_{+}}$и $\widetilde{\mathscr{F}}_{t}=\mathscr{F}_{T+t}$, ибо для $s \in \mathbf{R}_{+}$ $\left\{\widetilde{T}_{t} \leqslant s\right\}=\{t-T \leqslant s\} \in \mathscr{F}_{T} \subset \mathscr{F}_{T+t}=\widetilde{\mathscr{F}}_{t}$. Пусть теперь $(\Omega, \mathscr{F}, \widetilde{\mathfrak{G}}, \mathbf{P})$ с $\widetilde{\mathfrak{G}}=\left(\widetilde{\mathscr{G}}_{t}\right)_{t \in \mathbf{R}_{+}}$есть расширение стохастического базиса $(\Omega, \mathscr{F}, \widetilde{\mathfrak{F}}, \mathbf{P})($ без 
ограничения общности можно считать фильтрации $\widetilde{\mathfrak{F}}$ и $\widetilde{\mathfrak{G}}$ определенными на едином вероятностном пространстве $(\Omega, \mathscr{F}, \mathbf{P}))$. Определим для $t \in \mathbf{R}_{+} \sigma$-алгебру $\mathscr{G}_{t}$ событий из $\mathscr{F}$, пересечения которых с множеством $\{t<T\}$ принадлежат $\mathscr{F}_{t}$, а с множеством $\{t \geqslant T\}-\widetilde{\mathscr{G}}_{\widetilde{T}_{t}}$. Так как $\{T \leqslant t\} \in \mathscr{F}_{t}$ и $\mathscr{F}_{t} \subset \widetilde{\mathscr{F}}_{\widetilde{T}_{t}} \subset \widetilde{\mathscr{G}}_{\widetilde{T}_{t}}$, то $\mathscr{F}_{t} \subset \mathscr{G}_{t}$. Очевидно, фильтрация $\mathfrak{G}=\left(\mathscr{G}_{t}\right)_{t \in \mathbf{R}_{+}}$непрерывна справа. Докажем следующий результат.

Лемма. Пусть $(\Omega, \mathscr{F}, \widetilde{\mathfrak{G}}, \mathbf{P})$ является очень хорошим расширением для $(\Omega, \mathscr{F}, \widetilde{\mathfrak{F}}, \mathbf{P})$. Тогда $(\Omega, \mathscr{F}, \mathfrak{G}, \mathbf{P})$ является очень хорошим расширением для $(\Omega, \mathscr{F}, \mathfrak{F}, \mathbf{P})$.

Д о к а з а т е л ь с в о. Пусть $M$ - равномерно интегрируемый F-мартингал. Имеем тогда для $s \leqslant t$

$$
\begin{aligned}
\mathbf{E}\left(M_{t} \mid \mathscr{G}_{s}\right) & =1_{\{s<T\}} \mathbf{E}\left(M_{t} \mid \mathscr{G}_{s}\right)+1_{\{s \geqslant T\}} \mathbf{E}\left(M_{t} \mid \mathscr{G}_{s}\right) \\
& =1_{\{s<T\}} \mathbf{E}\left(M_{t} \mid \mathscr{G}_{s}\right)+1_{\{s \geqslant T\}} \mathbf{E}\left(M_{t} \mid \widetilde{\mathscr{G}}_{\widetilde{T}_{s}}\right) \\
& =1_{\{s<T\}} M_{s}+1_{\{s \geqslant T\}} \mathbf{E}\left(M_{T+\widetilde{T}_{t}} \mid \widetilde{\mathscr{G}}_{\widetilde{T}_{s}}\right) \\
& =1_{\{s<T\}} M_{s}+1_{\{s \geqslant T\}} M_{T+\widetilde{T}_{s}}=1_{\{s<T\}} M_{s}+1_{\{s \geqslant T\}} M_{s}=M_{s},
\end{aligned}
$$

что и означает, что $M$ есть $\mathfrak{G}$-мартингал.

Д о к а за т ел ь с т в о т е о р е м ы 2. Без ограничения общности, при необходимости расширяя вероятностное пространство $(\Omega, \mathscr{F}$, $\mathbf{P})$, можно считать, что существует равномерно распределенная на $[0,1]$ случайная величина $\xi$, не зависящая от $\mathscr{F}_{\infty}$. Отсюда, в свою очередь, следует существование последовательности равномерно распределенных на $[0,1]$ случайных величин $\xi_{1}, \xi_{2}, \ldots$, не зависяших друг от друга и от $\mathscr{F}_{\infty}$.

Итак, совершенно аналогично доказательству теоремы 1 , из уравнения (5) исключается процесс $N$. Далее, по теореме 1 существует очень хорошая решаюшая мера для уравнения (5) с $N \equiv 0$ (и соответственно преобразованной функцией $h$ ) и мерой $1_{D^{c} \times E} \cdot \theta$ вместо $\theta$. Ей отвечает, аналогично теореме 2.18 из [8] или предложению 9.16 из [10], решающий процесс $X^{(0)}$ этого уравнения на очень хорошем расширении $\left(\Omega, \mathscr{F}, \mathfrak{G}^{(1)}, \mathbf{P}\right)$ стохастического базиса $(\Omega, \mathscr{F}, \mathfrak{F}, \mathbf{P})$ с $\mathfrak{G}^{(1)}=\left(\mathscr{G}_{t}^{(1)}\right)_{t \in \mathbf{R}_{+}}$. Заметим теперь, что след бэровской $\sigma$-алгебры для тихоновской топологии на $D_{[0, \infty[}\left(\mathbf{R}^{d}\right)$ совпадает с борелевской $\sigma$-алгеброй на этом пространстве для $J_{1}$-топологии Скорохода, а борелевские множества для тихоновской топологии универсально измеримы относительно этой $\sigma$ алгебры. Поэтому в силу сепарабельности пространства $D_{[0, \infty[}\left(\mathbf{R}^{d}\right)$ с $J_{1}$-топологией Скорохода можно выбрать

$$
\mathscr{G}_{\infty}^{(1)}=\bigvee_{t \in \mathbf{R}_{+}} \mathscr{G}_{t}^{(1)} \subset \sigma\left(\mathscr{F}_{\infty}, \xi_{1}\right)
$$

Процесс $h\left(X^{(0)}\right) \times\left(g_{1} 1_{\left.\llbracket \tau_{1}\right] \times E} \cdot \theta\right)=h\left(X^{(0)}\right) 1_{\left[0, \tau_{1}\right] \times E} \times\left(g_{1} 1_{D \times E} \cdot \theta\right)$ - чисто скачкообразный с единственным скачком в момент $\tau_{1}$, и $h\left(X^{(0)}\right) \in$ 
$L^{1,2}\left(g_{1} 1_{\left.\llbracket \tau_{1}\right] \times E} \cdot \theta\right)$. Поэтому $h\left(X^{(0)}\right) \in L^{1,0}\left(1_{\left.\llbracket \tau_{1}\right] \times E} \cdot \theta\right)$, и процесс $h\left(X^{(0)}\right) \times$ $\left(1_{\left.\llbracket \tau_{1}\right] \times E} \cdot \theta\right)$ - чисто скачкообразный с единственным скачком в момент $\tau_{1}$, равным $\Delta\left(h\left(X^{(0)}\right) \times\left(g_{1} 1_{\llbracket \tau_{1} \rrbracket \times E} \cdot \theta\right)\right) / g_{1}$. Положим на $\llbracket 0, \tau_{1} \rrbracket$ процесс $X^{(1)}$ равным $X^{(0)}+h\left(X^{(0)}\right) \times\left(1_{\left.\llbracket \tau_{1}\right\rfloor \times E} \cdot \theta\right)$, тогда он является решающим для уравнения (5) на стохастическом интервале $\llbracket 0, \tau_{1} \rrbracket$.

Пусть теперь для $t \in \mathbf{R}_{+} \widetilde{\mathscr{F}}_{t}=\mathscr{G}_{t+\tau_{1}}^{(1)}$ и $\widetilde{\mathfrak{F}}=\left(\widetilde{\mathscr{F}}_{t}\right)_{t \in \mathbf{R}_{+}}, \widetilde{h}_{t}(\omega, \check{\omega}, u)=$ $h_{t+\tau_{1}}\left(\omega, \check{\omega}^{\left(X^{(1)}, \tau_{1}\right)}, u\right)$, где

$$
\omega_{t}^{\left(X^{(1)}, \tau_{1}\right)}= \begin{cases}X_{t}^{(1)}, & t<\tau_{1}, \\ \check{\omega}_{t+\tau_{1}}, & t \geqslant \tau_{1},\end{cases}
$$

и $\widetilde{\theta}_{t}\left(\varphi_{t, u}\right)=\widetilde{\varphi} \times \theta_{t+\tau_{1}}$, где

$$
\tilde{\varphi}_{t, u}= \begin{cases}0, & t \leqslant \tau_{1}, \\ \varphi_{t-\tau_{1}, u}, & t>\tau_{1},\end{cases}
$$

так что $\varphi \in L^{1,0}(\widetilde{\theta})$ (соответственно $\varphi \in \widehat{L}^{0}(\widetilde{\theta})$ ) тогда и только тогда, когда $\widetilde{\varphi} \in L^{1,0}(\theta)$ (соответственно $\widetilde{\varphi} \in \widehat{L}^{0}(\theta)$ ). Рассмотрим уравнение

$$
X=X_{\tau_{1}}^{(1)}+\tilde{h}(X) \times \tilde{\theta} .
$$

Тогда оно, рассматриваемое на стохастическом базисе $(\Omega, \mathscr{F}, \widetilde{\mathfrak{F}}, \mathbf{P})$, удовлетворяет условиям теоремы 2 с моментами $\tau_{2}-\tau_{1}, \tau_{3}-\tau_{1}, \ldots$ вместо $\tau_{1}, \tau_{2}, \ldots$ и существует решающий процесс $\widetilde{X}^{(2)}$ для уравнения $(6)$ на стохастическом интервале $\llbracket 0, \tau_{2}-\tau_{1} \rrbracket$ на очень хорошем расширении $\left(\Omega, \mathscr{F}, \widetilde{\mathfrak{G}}^{(2)}, \mathbf{P}\right)$ с $\widetilde{\mathfrak{G}}^{(2)}=\left(\tilde{\mathscr{G}}_{t}^{(2)}\right)_{t \in \mathbf{R}_{+}}$стохастического базиса $(\Omega, \mathscr{F}, \widetilde{\mathfrak{F}}, \mathbf{P})$, причем можно выбрать $\widetilde{\mathscr{G}}_{\infty}^{(2)}=\bigvee_{t \in \mathbf{R}_{+}} \tilde{\mathscr{G}}_{t}^{(2)} \subset \sigma\left(\mathscr{G}_{\infty}^{(1)}, \xi_{2}\right)$. Определим процесс $X^{(2)}$ равенствами

$$
X_{t}^{(2)}= \begin{cases}X_{t}^{(1)}, & t<\tau_{1}, \\ \widetilde{X}_{t-\tau_{1}}^{(2)}, & t \geqslant \tau_{1} .\end{cases}
$$

Тогда он является решающим процессом уравнения (5) на стохастическом базисе $\left(\Omega, \mathscr{F}, \mathfrak{G}^{(2)}, \mathbf{P}\right)$ (который строится из $\left(\Omega, \mathscr{F}, \tilde{\mathfrak{G}}^{(2)}, \mathbf{P}\right)$ в соответствии с леммой и с моментом $\tau_{1}$ вместо $T$ ) и стохастическом интервале $\left[0, \tau_{2} \rrbracket\right.$.

Итак, для всякого $n \in \mathbf{N}$ мы имеем фильтрацию $\mathfrak{G}^{(n)}=\left(\mathscr{G}_{t}^{(n)}\right)_{t \in \mathbf{R}_{+}}$, с которой стохастический базис $\left(\Omega, \mathscr{F}, \mathfrak{G}^{(n)}, \mathbf{P}\right)$ является очень хорошим расширением стохастического базиса $(\Omega, \mathscr{F}, \mathfrak{F}, \mathbf{P})$, и процесс $X^{(n)}$, который на стохастическом интервале $\llbracket 0, \tau_{n} \rrbracket$ является решающим процессом уравнения (5) на стохастическом базисе $\left(\Omega, \mathscr{F}, \mathfrak{G}^{(n)}, \mathbf{P}\right)$, причем для $m<n \mathscr{G}_{t}^{(m)} \subset \mathscr{G}_{t}^{(n)}$ при всех $t \in \mathbf{R}_{+}$и сужение $X^{(n)}$ на $\llbracket 0, \tau_{m} \rrbracket$ совпадает с $X^{(m)}$. В силу последнего, так как $\mathbf{P}$-п.н. $\lim _{n \rightarrow \infty} \tau_{n}=\infty$, существует процесс $X$, сужение которого на $\llbracket 0, \tau_{n} \rrbracket$ совпадает с $X^{(n)}$. Обозначим теперь $\mathscr{G}_{t}=\bigvee_{n=1}^{\infty} \mathscr{G}_{t}^{(n)}$ и $\mathfrak{G}=\left(\mathscr{G}_{t}\right)_{t \in \mathbf{R}_{+}}$. Так как для каждого 
$t \in \mathbf{R}_{+} \sigma$-алгебра $\mathscr{G}_{t}$ состоит из событий, пересечения которых с событием $\left\{t<\tau_{1}\right\}$ принадлежат $\mathscr{G}_{t}^{(1)}$, а с событиями $\left\{\tau_{n-1} \leqslant t<\tau_{n}\right\}-$ соответственно $\mathscr{G}_{t}^{(n)}$, то фильтрация $\mathfrak{G}$ непрерывна справа, и, аналогично лемме, $(\Omega, \mathscr{F}, \mathfrak{G}, \mathbf{P})$ является очень хорошим расширением стохастического базиса $(\Omega, \mathscr{F}, \mathfrak{F}, \mathbf{P})$, на котором $X$ является решающим процессом уравнения (5), и ему отвечает очень хорошая решающая мера. Теорема доказана.

3 а м е ч а н и е 1 . Теорема 2 остается справедливой, если в качестве $D$ взять дискретное $\overline{\mathfrak{F}}$-опциональное множество из графиков $\overline{\mathfrak{F}}$-моментов остановки $\tau_{n}(\omega, \check{\omega})$, для которого $1_{D^{c} \times E} \cdot \theta \in \widetilde{\mathscr{S}}_{\sigma}^{2}(\widetilde{\mathbf{P}})$ и $g_{n} 1_{\llbracket \tau_{n} \rrbracket \times E} \cdot \theta \in$ $\widetilde{\mathscr{S}}_{\sigma}^{2}(\widetilde{\mathbf{P}})$ со строго положительными $\overline{\mathscr{F}}_{\tau_{n}}$-измеримыми случайными величинами $g_{n}$ при любой вероятностной мере $\widetilde{\mathbf{P}}$ на $(\bar{\Omega}, \bar{F}), \Omega$-маргинал которой равен $\mathbf{P}$ и для которой $(\bar{\Omega}, \overline{\mathscr{F}}, \overline{\mathfrak{F}}, \widetilde{\mathbf{P}})$ является очень хорошим расширением стохастического базиса $(\Omega, \mathscr{F}, \mathfrak{F}, \mathbf{P})$. Действительно, если $X^{(0)}$ — решающий процесс уравнения (5) с $N \equiv 0$ и мерой $1_{D^{c} \times E} \cdot \theta$ вместо $\theta$ на очень хорошем расширении $\left(\Omega, \mathscr{F}, \mathfrak{G}^{(1)}, \mathbf{P}\right)$ стохастического базиса $(\Omega, \mathscr{F}, \mathfrak{F}, \mathbf{P})$, то $\tau_{1}\left(\omega, X^{(0)}\right)$ является $\mathfrak{G}^{(1)}$-моментом остановки, и все дальнейшее проходит аналогично доказательству теоремы 2.

3 а м е ч а н и е 2. Если для некоторой возрастающей к $\infty$ (необязательно $\mathbf{P}$-п.н. строго) последовательности $\left(\tau_{n}\right)_{n \in \mathbf{N}}$ моментов остановки относительно $\mathfrak{F}$ (или $\overline{\mathfrak{F}}$ ) выполнены условия теоремы 1 для мер $1_{\left[0, \tau_{n}[\times E\right.} \cdot \theta$ вместо $\theta$, то выполнены и условия теоремы 2 (с учетом замечания 1 для $\overline{\mathfrak{F}})$. Действительно, из условий теоремы 1 вытекает локальная принадлежность функции $h$ при разных $\check{\omega}$ к ограниченному множеству в $L^{1,2}(\theta)$ (которую легкко сделать глобальной надлежащим выбором моментов остановки $\tau_{n}$ ), и тогда случайную величину $g_{1}$ можно выбрать принимающей постоянные значения на множествах вида $\llbracket\left[\tau_{1} \rrbracket \cap \llbracket 0, \tau_{n} \llbracket\right.$, и далее рассуждение проходит аналогично доказательству теоремы 2.

3. Основные примеры. Приведем теперь примеры, демонстрирующие возможность сведения к теоремам 1 и 2 прежних результатов о существовании слабых решений стохастических дифференциальных уравнений.

П р и м е р 1. Теорема 9.20 из [10] является частным случаем теоремы 1 с множеством $\bar{E}=E \cup\{1, \ldots, 2 r\}$ вместо $E$, в котором точки $1, \ldots, 2 r$ изолированы от $E$, с

$$
\theta_{t}(\varphi)=\sum_{i=1}^{r} \varphi(i) \cdot a_{t}^{i}+\sum_{i=1}^{r} \varphi(r+i) \cdot \bar{m}_{t}^{i}+\varphi *\left(\mu^{c}-\nu^{c}\right)_{t}+\varphi * \mu_{t}^{d}
$$

где $\bar{m}^{i}$ - процессы из доказательства теоремы 9.20 в [10], получаемые ортогонализацией системы $\left(m^{1}, \ldots, m^{r}\right)$ с $\bar{m}=T \cdot m$, а последний стохастический интеграл понимается в семимартингальном смысле, и с функ- 
цией $\widehat{h}$ вместо $h$, равной

$$
\widehat{h}= \begin{cases}f^{u}, & u=1, \ldots, r, \\ g^{u-r}, & u=r+1, \ldots, 2 r, \text { c } \bar{g}=g T^{T}, \\ h, & u \in E .\end{cases}
$$

Очевидно, $\theta \in \widetilde{\mathscr{S}}_{\sigma}^{2}$, причем в качестве $X^{1}$ можно взять процесс $B$, фигурирующий в условиях теоремы 9.20 из [10] и пригодный в качестве $B$ и в условиях теоремы 1 , в качестве $X^{3 i-1}$ для $i=1, \ldots, r-$ процессы $\bar{m}^{i}$, а для $i>r$ их можно взять тождественно равными нулю, в качестве $X^{3 i}$ - процессы вида $\varphi^{3 i} *\left(\mu^{c}-\nu^{c}\right)$ с $\varphi^{3 i} \varphi^{3 j} * \nu^{c} \equiv 0$ для $i \neq j$, дающие полное ортогональное разложение стохастических интегралов вида $\varphi *\left(\mu^{c}-\nu^{c}\right)$, в качестве $X^{3 i+1}-$ процессы вида $\varphi^{3 i+1} *\left(\mu^{d}-\nu^{d}\right)$ с $\left(\varphi^{3 i+1}-\bar{\varphi}^{3 i+1}\right)\left(\varphi^{3 j+1}-\bar{\varphi}^{3 j+1}\right) * \nu^{d} \equiv 0$ для $i \neq j$, дающие полное ортогональное разложение стохастических интегралов вида $\varphi *\left(\mu^{d}-\nu^{d}\right)$. Если имеются факторизации $d a_{t}^{j}(\omega)=A_{t}^{j}(\omega) d B_{t}(\omega)$,

$$
\begin{aligned}
d\left\langle m^{j}, m^{l}\right\rangle(\omega) & =M_{t}^{j l}(\omega) d B_{t}(\omega), \quad d\left\langle\bar{m}^{j}, \bar{m}^{j}\right\rangle(\omega)=\bar{M}_{t}^{j}(\omega) d B_{t}(\omega), \\
\nu^{c}(\omega, d t \times d u) & =\rho_{t}^{c}(\omega, d u) d B_{t}(\omega), \quad \nu^{d}(\omega, d t \times d u)=\rho_{t}^{d}(\omega, d u) d B_{t}(\omega),
\end{aligned}
$$

в терминах которых формулируются условия теоремы 9.20 из [10], то мера $\theta$ имеет разложение вида $(3)$ с $\rho_{t}^{1}(\{j\})=A^{j}$ для $1 \leqslant j \leqslant r$, $\rho_{t}^{1}(\{j\})=0$ для $r+1 \leqslant j \leqslant 2 r, \rho^{1}=\rho^{d}$ на $E$, при $1 \leqslant i \leqslant r \rho_{t}^{3 i-1}(\{j\})=0$ для $1 \leqslant j \leqslant r, \rho_{t}^{3 i-1}(\{j\})=1$ для $r+1 \leqslant j \leqslant 2 r, \rho^{3 i-1}=0$ на $E$, при $i>r$ $\rho^{3 i-1} \equiv 0, \rho_{t}^{3 i}(\{j\})=0$ для $1 \leqslant j \leqslant 2 r$,

$$
\rho_{t}^{3 i}(\Gamma)=\frac{\int_{\Gamma} \varphi_{t, u}^{3 i} \rho_{t}^{c}(d u)}{\int_{E}\left(\varphi_{t, u}^{3 i}\right)^{2} \rho_{t}^{c}(d u)} \quad \text { для } \Gamma \in \mathscr{B}(E),
$$

$\rho_{t}^{3 i+1}(\{j\})=0$ для $1 \leqslant j \leqslant 2 r$,

$$
\rho_{t}^{3 i+1}(\Gamma)=\frac{\int_{\Gamma}\left(\varphi_{t, u}^{3 i+1}-\bar{\varphi}_{t, u}^{3 i+1}\right) \rho_{t}^{d}(d u)}{\int_{E}\left(\varphi_{t, u}^{3 i+1}-\bar{\varphi}_{t, u}^{3 i+1}\right)^{2} \rho_{t}^{d}(d u)} \quad \text { для } \Gamma \in \mathscr{B}(E) .
$$

Имеем тогда (для матрицы $\bar{M}$ с элементами $\bar{M}^{j}$ на главной диагонали и 0 вне ее)

$$
\begin{aligned}
& \left|\int_{\bar{E}} \widehat{h} \rho^{1}(d u)\right| \frac{d X^{1}}{d B}=\left|\int_{\bar{E}} \widehat{h} \rho^{1}(d u)\right|=|f A|, \\
& \sum_{i=1}^{\infty}\left|\int_{\bar{E}} \widehat{h} \rho^{3 i-1}(d u)\right|^{2} \frac{d\left\langle X^{3 i-1}, X^{3 i-1}\right\rangle}{d B}=\sum_{i=1}^{r}\left|\bar{g}^{i}\right|^{2}\left(\bar{M}^{i}\right)^{2} \\
& =\operatorname{Tr}\left(\bar{g} \bar{M} \bar{g}^{T}\right)=\operatorname{Tr}\left(g T^{T} \bar{M} T g^{T}\right)=\operatorname{Tr}\left(g N g^{T}\right), \\
& \sum_{i=1}^{\infty}\left|\int_{\bar{E}} \widehat{h} \rho^{3 i}(d u)\right|^{2} \frac{d\left\langle X^{3 i}, X^{3 i}\right\rangle}{d B}=\sum_{i=1}^{\infty} \sum_{k=1}^{d}\left(\int_{\bar{E}} \widehat{h}^{k} \rho^{3 i}(d u)\right)^{2} \frac{d\left\langle X^{3 i}, X^{3 i}\right\rangle}{d B} \\
& =\sum_{k=1}^{d} \sum_{i=1}^{\infty} \frac{\left(\int_{E} h^{k} \varphi^{3 i} \rho^{c}(d u)\right)^{2}}{\int_{E}\left(\varphi^{3 i}\right)^{2} \rho^{c}(d u)}=\sum_{k=1}^{d} \int_{E}\left(h^{k}\right)^{2} \rho^{c}(d u)=\int_{E}|h|^{2} \rho^{c}(d u)
\end{aligned}
$$


в силу ортогональности относительно $\rho^{c}$ разложения функций $h^{k}$ по $\varphi^{3 i}$, а также

$$
\begin{aligned}
& \sum_{i=1}^{\infty}\left|\int_{\bar{E}} \hat{h} \rho^{3 i+1}(d u)\right|^{2} \frac{d\left\langle X^{3 i+1}, X^{3 i+1}\right\rangle}{d B} \\
& \quad=\int_{E}|h-\bar{h}|^{2} \rho^{d}(d u)=\int_{E}|h|^{2} \rho^{d}(d u)-\frac{\left|\int_{E} h \rho^{d}(d u)\right|^{2}}{\Delta B}
\end{aligned}
$$

так что в силу условия (1) теоремы 9.20 из [10] выполнено условие 1) теоремы 1. Непрерывность в условии 2) теоремы 1 вытекает очевидным образом из условия непрерывности (2) теоремы 9.20 из [10].

Именно, $\int_{\bar{E}} \widehat{h} \rho^{1}(d u)=f A+\int_{E} h \rho^{d}(d u)$, причем $\int_{E} \rho^{d}(d u)=1 / \Delta B$ и $\int_{E}|h|^{2} \rho^{d}(d u) \leqslant c+\left|\int_{E} h \rho^{d}(d u)\right|^{2} \Delta B \leqslant c+c^{2} \Delta B$, так что $h$ равномерно $\rho^{d}$-интегрируема на $E, \int_{\bar{E}} \widehat{h} \rho^{3 i-1}(d u)=\bar{g}^{i}$ для $1 \leqslant i \leqslant r, \int_{\bar{E}} \widehat{h} \rho^{3 i-1}(d u)=0$ для $i>r$,

$$
\int_{\bar{E}} \widehat{h} \rho^{3 i}(d u)=\frac{\int_{E} h \varphi^{3 i} \rho^{c}(d u)}{\int_{E}\left(\varphi^{3 i}\right)^{2} \rho^{c}(d u)}
$$

и

$$
\int_{E}|h|\left|\varphi^{3 i}\right| \rho^{c}(d u) \leqslant\left(\int_{E}\left(\varphi^{3 i}\right)^{2} \rho^{c}(d u)\right)^{1 / 2}\left(\int_{E}|h|^{2} \rho^{c}(d u)\right)^{1 / 2},
$$

так что $h \varphi^{3 i}$ равномерно $\rho^{c}$-интегрируема на $E$,

$$
\int_{\bar{E}} \widehat{h} \rho^{3 i+1}(d u)=\frac{\int_{E} h\left(\varphi^{3 i+1}-\bar{\varphi}^{3 i+1}\right) \rho^{d}(d u)}{\int_{E}\left(\varphi^{3 i+1}-\bar{\varphi}^{3 i+1}\right)^{2} \rho^{d}(d u)}
$$

и точно так же $h \varphi^{3 i+1}$ равномерно $\rho^{d}$-интегрируема на $E$.

В условии 2) равномерная сходимость ряда

$$
\sum_{i=1}^{\infty}\left|\int_{\bar{E}} \widehat{h} \rho^{3 i-1}(d u)\right|^{2} \frac{d\left\langle X^{3 i-1}, X^{3 i-1}\right\rangle}{d B}
$$

следует тривиально из его конечности, а равномерная сходимость ряда

$$
\sum_{i=1}^{\infty}\left|\int_{\bar{E}} \widehat{h} \rho^{3 i}(d u)\right|^{2} \frac{d\left\langle X^{3 i}, X^{3 i}\right\rangle}{d B}
$$

- из $U$-непрерывности его членов и суммы, равной $\int_{E}|h|^{2} \rho^{c}(d u)$, следующей, в свою очередь, из непрерывности функции $|h|^{2}$ и ее равномерной $\rho^{c}$-интегрируемости.

Наконец, требуемая в условии 3) равномерная интегрируемость следует из равномерной интегрируемости в условии (3) теоремы 9.20 из [10].

П р и м е р 2. Пусть теперь выполнены условия теоремы 9.32 из [10]. Определим после исключения из уравнения (5) процесса $N \overline{\mathfrak{F}}$ моменты остановки $\tau_{n}(\omega, \check{\omega})=\inf \left\{t:\left|\check{\omega}_{t}\right|>n\right\}$. Так как почти наверное по любой решающей мере $\left|h_{t, u}(\omega, \check{\omega})\right| \leqslant 2 n$ на множестве $\llbracket 0, \tau_{n} \llbracket \times E$, то на каждом таком множестве выполнены условия теоремы 9.20 из [10], которая в силу примера 1 сводится к теореме 1 , и с учетом замечаний 2 и 1 выполнены условия теоремы 2 . 


\section{СПИСОК ЛИТЕРАТУРЫ}

1. Bichteler K., Jacod J. Random measures and stochastic integration. - Lecture Notes in Control and Inform. Sci., 1983, v. 49, p. 1-18.

2. Лебедев $B$. A. Поведение случайных мер при замене фильтрации. - Теория вероятн. и ее примен., 1995 , т. 40 , в. 4 , с. $754-763$.

3. Lebedev $V$. A. On the existence of weak solutions for stochastic differential equations with driving $L^{2}$-valued measures. - Международная конференция «Асимптотические методы в теории вероятностей и математической статистике», посвященная 50-летию образования кафедры теории вероятностей и математической статистики С.-Петербургского государственного университета. Тезисы докладов. С.Петербург, 1998, с. 151-155.

4. Eméry $M$. Une topologie sur l'espace des semimartingales. - Lecture Notes in Math., 1979 , v. 721 , p. $260-280$.

5. Лебедев $B$. A. Теорема Фубини для зависящих от параметра стохастических интегралов по $L^{0}$-значным случайным мерам. - Теория вероятн. и ее примен., 1995 , т. 40 , в. 2 , с. $313-323$.

6. Lebedev V. A. On infinite-dimensional stochastic integration. - Statistics and Control of Stochastic Processes: Steklov Seminar 1984. New York: Optimization Software, 1989, p. 277-304.

7. Деллашери К. Емкости и случайные процессы. М.: Мир, 1975, 192 с.

8. Jacod J., Mémin J. Weak and strong solutions of stochastic differential equations: existence and stability. - Lecture Notes in Math., 1981, v. 851, p. 169-212.

9. Lebedev $V$. A. On the existence of weak solutions for stochastic differential equations with driving martingales and random measures. - Stochastics, 1983, v. 9, № 1-2, p. 37-76.

10. Лебедев В. А. Мартингалы, сходимость вероятностных мер и стохастические уравнения. - М.: Изд-во МАИ, 1996, 348 с.

11. Ширяев А. Н. Вероятность. М.: Наука, 1989, 640 с.

12. Лебедев B. А. О существовании слабых решений для стохастических дифференциальных уравнений с ведущими $L^{0}$-значными мерами. - Обозрение прикл. и промышл. матем., сер. вероятн. статист., 1998, т. 5, в. 2, с. 246.

Поступила в редакцию 26.III. 1999

Исправленный вариант 23.V.2000 